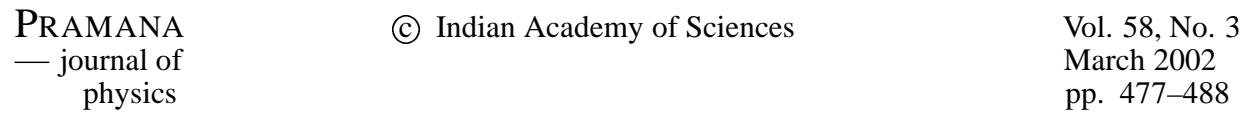

\title{
Invariant metric for nonlinear symplectic maps
}

\author{
GOVINDAN RANGARAJAN ${ }^{* \dagger}$ and MINITA SACHIDANAND* \\ * Department of Mathematics, Indian Institute of Science, Bangalore 560 012, India \\ ${ }^{\dagger}$ Also at Centre for Theoretical Studies, Indian Institute of Science, Bangalore, India \\ $\ddagger$ Jawaharlal Nehru Centre for Advanced Scientific Research, Bangalore, India \\ Email: rangaraj@math.iisc.ernet.in
}

MS received 6 June 2001

\begin{abstract}
In this paper, we construct an invariant metric in the space of homogeneous polynomials of a given degree $(\geq 3)$. The homogeneous polynomials specify a nonlinear symplectic map which in turn represents a Hamiltonian system. By minimizing the norm constructed out of this metric as a function of system parameters, we demonstrate that the performance of a nonlinear Hamiltonian system is enhanced.
\end{abstract}

Keywords. Invariant metric; symplectic maps; performance optimization.

PACS Nos 05.45.-a; 02.20.-a; 45.20.Jj; 41.85.-p

\section{Introduction}

One popular method of treating Hamiltonian systems perturbatively is the Lie algebraic method [1-5]. In this approach, the time evolution of a Hamiltonian system is described by a symplectic map $\mathscr{M}$ [1]. This map is specified by a set of homogeneous polynomials of degree $m$. The polynomials of degree greater than or equal to 3 give the nonlinear content of $\mathscr{M}$. For a generic nonlinear Hamiltonian system, the full symplectic map can not be explicitly computed. This forces us to follow the Lie perturbative approach where we consider terms degree by degree in the symplectic map. Now if we are able to construct a norm for the space of homogeneous polynomials of a given degree, it can be used to quantify the nonlinear content of $\mathscr{M}$. This has several applications described later.

In order to obtain such a norm, we first construct a bilinear form on the space of homogeneous polynomials of degree $m$ that is both symmetric and positive definite. This defines a metric on this space which then enables us to construct a norm. Further, the norm should be invariant under the appropriate symmetry group. Thus, we define an invariant metric to be a symmetric, positive definite, bilinear form that is invariant under the action of a suitable symmetry group. The underlying symmetry group of the symplectic map $\mathscr{M}$ is an infinite dimensional non-compact Lie group. Fortunately, we can restrict ourselves to a much smaller symmetry group for the following reason. It turns out that the symplectic map $\mathscr{M}$ can be represented in terms of homogeneous polynomials as 


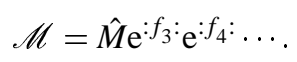

The meaning of each term above will be explained in greater detail in the next section. For the present we note that $f_{m}$ is a homogeneous polynomial of degree $m$ in $z$. It turns out that $\mathrm{e}^{: f_{3}:}$ contains the leading order nonlinearity of the symplectic map $\mathscr{M}$. Therefore, in the spirit of perturbation theory we can truncate our map as follows:

$$
\mathscr{M}=\hat{M} \mathrm{e}^{: f_{3}:} \text {. }
$$

Suppose we have a norm on the space of homogeneous polynomials of degree 3 . Then the norm of the polynomial $f_{3}$ quantifies the leading order nonlinearity of $\mathscr{M}$. Now $f_{3}$ is a function of the parameters specifying the Hamiltonian under consideration and so obviously is its norm. Therefore, minimizing the norm of $f_{3}$ as a function of one or more of these parameters corresponds to minimizing the leading order nonlinearity of $\mathscr{M}$. By using the optimal value(s) of the parameter(s) thus obtained in the original Hamiltonian system, one can obtain substantial improvements in the performance of most nonlinear Hamiltonian systems (especially in increasing the stability region). Furthermore, the norm can also serve as a measure to compute the 'distance' between two symplectic maps.

Now the symmetry group for the linear part $\hat{M}$ for $n$ degrees of freedom is the finite dimensional non-compact real symplectic group $\operatorname{Sp}(2 n, R)$. A norm that is invariant under the linear part would also be invariant under $\mathscr{M}=\hat{M} \mathrm{e}^{:} f_{3}:$ since the nonlinear term contributes only a fourth order correction. Therefore, the relevant symmetry group for our purpose would be $\operatorname{Sp}(2 n, R)$. However, as $\operatorname{Sp}(2 n, R)$ is non-compact, there can be no norm that is invariant under the action of this group. To get around this problem, we first convert $\hat{M}$ into its so-called 'normal form' [1] using a symplectic transformation. Once this transformation is made, we can take the relevant symmetry group to be the compact group $\mathrm{SU}(n)[4,5]$. Thus, our problem reduces to finding norms for the space of homogeneous polynomials of degree 3 in the $2 n$ phase space variables invariant under the action of $\mathrm{SU}(n)$. This is accomplished by performing an invariant integration over $\mathrm{SU}(n)$ of a suitable function. Finally, we apply the above procedure to a FODO lattice (a common component of a particle accelerator system). We demonstrate that minimizing the norm leads to significant improvements in the stability region of this system.

\section{Preliminaries}

In this section, we briefly review the basic mathematical results required for the remaining part of the paper.

We start by representing a Hamiltonian system by a symplectic map [1]. Let us denote the collection of $2 n$ phase-space variables $q_{i}, p_{i}(i=1,2, \ldots, n)$ by the symbol $z$ :

$$
z=\left(q_{1}, q_{2}, \ldots, q_{n}, p_{1}, p_{2}, \ldots, p_{n}\right) .
$$

The Lie operator [1] corresponding to a phase-space function $f(z)$ is denoted by $: f(z):$. It is defined by its action on a phase-space function $g(z)$ as shown below

$$
: f(z): g(z)=[f(z), g(z)]
$$

Here $[f(z), g(z)]$ denotes the usual Poisson bracket of the functions $f(z)$ and $g(z)$. Next, we define the exponential of a Lie operator. It is called a Lie transformation [1] and is given as follows: 


$$
\mathrm{e}^{: f(z):}=\sum_{n=0}^{\infty} \frac{: f(z):^{n}}{n !} .
$$

The effect of a Hamiltonian system on a particle can be formally expressed as the action of a map $\mathscr{M}$ that takes the particle from its initial state $z^{\text {in }}$ to its final state $z^{\text {fin }}$

$$
z^{\text {fin }}=\mathscr{M} z^{\text {in }} .
$$

It can be shown that $\mathscr{M}$ is a symplectic map [1]. Symplectic maps are maps whose $2 n \times 2 n$ Jacobian matrices $M(z)$ satisfy the following 'symplectic condition'

$$
\widetilde{M(z)} J M(z)=J
$$

where $\tilde{M}$ is the transpose of $M$ and $J$ is the fundamental symplectic matrix. The set of all symplectic matrices forms the real symplectic group $\operatorname{Sp}(2 n, R)$.

Using the Dragt-Finn factorization theorem [6], the symplectic map $\mathscr{M}$ can be factorized as shown below:

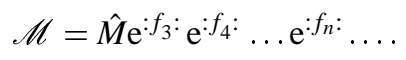

Here $\hat{M}$ gives the linear part of the map and hence has an equivalent representation in terms of the Jacobian matrix $M$ of the map $\mathscr{M}[1]$ :

$$
\hat{M} z_{i}=M_{i j} z_{j}=(M z)_{i}
$$

The infinite product of Lie transformations $\exp \left(: f_{n}:\right)(n=3,4, \ldots)$ in eq. (2.6) represents the nonlinear part of $\mathscr{M}$ where $f_{n}(z)$ denotes a homogeneous polynomial (in $z$ ) of degree $n$ uniquely determined by the factorization theorem.

From the above discussion we see that homogeneous polynomials play an important role in Lie perturbation theory. In particular, they represent the nonlinear content of the symplectic map (and hence the underlying Hamiltonian system). To facilitate construction of an invariant metric on the space of homogeneous polynomials, we first need to index the basis monomials appropriately. Consider the space $\mathscr{P}(m)$ of all homogeneous polynomials in $z$ of degree $m$. Let $\left\{P_{\alpha}^{(m)}\right\}$ be the basis for this space. The dimension $N(2 n, m)$ of this space is [7]

$$
N(2 n, m)=\left(\begin{array}{c}
2 n+m-1 \\
m
\end{array}\right)
$$

We take the basis $P_{\alpha}^{(m)}(z)$ to be the $m$ th degree basis monomial in $2 n$ variables, i.e.,

$$
P_{\alpha}^{(m)}(z)=q_{1}^{r_{1}} p_{1}^{r_{2}} \ldots q_{n}^{r_{2 n-1}} p_{n}^{r_{2 n}}, \quad i \leq r_{i} \leq m, r_{1}+r_{2}+\cdots+r_{2 n}=m
$$

We can associate each basis monomial with a convenient numerical index $i$ [8] as shown below. Let

$$
i\left(r_{1}, r_{2}, \ldots, r_{2 n}\right)=\sum_{l=1}^{2 n}\left(\begin{array}{c}
l-1+\sum_{k=0}^{l-1} r_{2 n-k} \\
l
\end{array}\right)
$$


It can be easily shown that $p_{n}^{m}$ is the last monomial with degree less than or equal to $m$ in this indexing, i.e.,

$$
i(0,0, \ldots, 0, m)=\max _{r_{1}, r_{2}, \cdots, r_{2 n}} i\left(r_{1}, r_{2}, \ldots, r_{2 n}\right), \quad r_{1}+r_{2}+\cdots+r_{2 n} \leq m
$$

Thus, we see that $i(0,0, \ldots, 0, m)$ gives the total number of monomials less than or equal to $m$. Therefore, a convenient index $\alpha$ for the elements of the set $\left\{P_{\alpha}^{(m)}\right\}$ is got using (2.10) and (2.11). This is given by the relation

$$
\alpha\left(r_{1}, r_{2}, \ldots, r_{2 n}\right)=i\left(r_{1}, r_{2}, \ldots, r_{2 n}\right)-i(0,0, \ldots, 0, m-1)
$$

Thus, any polynomial $f_{m}(z)$ can be written in the following way:

$$
f_{m}(z)=a_{\alpha}^{(m)} P_{\alpha}^{(m)}(z)
$$

The quantities $a_{\alpha}^{(m)}$ are real constants.

From our discussion in the introduction, we know that $\mathrm{SU}(n)$ plays an important role in the construction of an invariant metric. We are most interested in single particle dynamics (with three degrees of freedom) for which the relevant group is SU(3). Since the behaviour of the system in its two transverse degrees of freedom is often quite useful to analyse, SU(2) is also relevant. We will obtain the invariant metric by performing an invariant integration over the above groups. Therefore, we now give the parameterizations for SU(2) and SU(3) along with their Haar measures that will be required for this invariant integration.

A parameterization for a general element $U$ of $\mathrm{SU}(2)$ is [9]

$$
U=\left(\begin{array}{cc}
\sin \theta_{21} \exp \left(-i \phi_{22}\right) & -\cos \theta_{21} \exp \left(-i \phi_{21}\right) \\
\cos \theta_{21} \exp \left(i \phi_{21}\right) & \sin \theta_{21} \exp \left(i \phi_{22}\right)
\end{array}\right)
$$

where

$$
0 \leq \theta_{21} \leq \frac{1}{2} \pi, \quad 0 \leq \phi_{21} \leq 2 \pi, \quad 0 \leq \phi_{22} \leq 2 \pi
$$

A parameterization for a general element $U$ of $\mathrm{SU}(3)$ is [10]

$$
U=\left(\begin{array}{ccc}
\sin \theta_{21} \sin \theta_{31} \mathrm{e}^{-i \phi_{22}} & \Gamma_{12} & \Gamma_{13} \\
\cos \theta_{21} \sin \theta_{31} \mathrm{e}^{i \phi_{21}} & \Gamma_{22} & \Gamma_{23} \\
\cos \theta_{31} \mathrm{e}^{i \phi_{31}} & \sin \theta_{31} \cos \theta_{32} \mathrm{e}^{i \phi_{32}} & \sin \theta_{31} \sin \theta_{32} \mathrm{e}^{i \phi_{33}}
\end{array}\right)
$$

with

$$
\begin{aligned}
& \Gamma_{12}=-\cos \theta_{21} \sin \theta_{32} \mathrm{e}^{i\left(-\phi_{21}-\phi_{33}\right)}-\sin \theta_{21} \cos \theta_{31} \cos \theta_{32} \mathrm{e}^{i\left(-\phi_{22}-\phi_{31}+\phi_{32}\right)}, \\
& \Gamma_{22}=\sin \theta_{21} \sin \theta_{32} \mathrm{e}^{i\left(\phi_{22}-\phi_{33}\right)}-\cos \theta_{21} \cos \theta_{31} \cos \theta_{32} \mathrm{e}^{i\left(\phi_{21}-\phi_{31}+\phi_{32}\right)}, \\
& \Gamma_{13}=\cos \theta_{21} \cos \theta_{32} \mathrm{e}^{i\left(-\phi_{21}-\phi_{32}\right)}-\sin \theta_{21} \cos \theta_{31} \sin \theta_{32} \mathrm{e}^{i\left(-\phi_{22}-\phi_{31}+\phi_{33}\right)}, \\
& \Gamma_{23}=-\sin \theta_{21} \cos \theta_{32} \mathrm{e}^{i\left(\phi_{22}-\phi_{32}\right)}-\cos \theta_{21} \cos \theta_{31} \sin \theta_{32} \mathrm{e}^{i\left(\phi_{21}-\phi_{31}+\phi_{33}\right)}
\end{aligned}
$$

Next, we specify the Haar measure for SU(2) and SU(3). The measure for SU(2) with parameterization (2.13) is [9] 


$$
\frac{1}{4 \pi^{2}} \sin \left(2 \theta_{21}\right) \mathrm{d} \theta_{21} \mathrm{~d} \phi_{21} \mathrm{~d} \phi_{22} \text {. }
$$

The case of SU(3) is more complicated, but following the procedure in [10] we have the invariant measure for $\mathrm{SU}(3)$ (using the parameterization given in (2.14)) to be

$$
\frac{1}{(2 \pi)^{5}} \sin \left(2 \theta_{21}\right) 4 \sin ^{3}\left(\theta_{31}\right) \cos \left(\theta_{31}\right) \sin \left(2 \theta_{32}\right) \mathrm{d} \theta_{21} \mathrm{~d} \theta_{31} \mathrm{~d} \theta_{32} \mathrm{~d} \phi_{21} \mathrm{~d} \phi_{22} \mathrm{~d} \phi_{31} \mathrm{~d} \phi_{32} \mathrm{~d} \phi_{33} .
$$

\section{Invariant metric}

For our purposes, we define an invariant metric to be a symmetric, positive definite, bilinear form on the space of homogeneous polynomials of a given degree $m$, in $2 n$ phase-space variables, that is invariant under the action of $\mathrm{SU}(\mathrm{n})$. This metric defines an inner product on the space of homogeneous polynomials of degree $m$. This in turn enables us to define an invariant norm on that space. This norm is used to quantify the nonlinear content of $\mathscr{M}$.

Our task now is to generate the invariant metric. Let $\mathscr{G}$ denote either SU(2) or SU(3). We define the bilinear form to be

$$
g_{\alpha \beta}^{(m)} \equiv\left(P_{\alpha}^{(m)}(z), P_{\beta}^{(m)}(z)\right) .
$$

We require $g_{\alpha \beta}^{(m)}$ to be invariant under the action of $\mathscr{G}$. That is,

$$
\left(U P_{\alpha}^{(m)}(z), U P_{\beta}^{(m)}(z)\right)=\left(P_{\alpha}^{(m)}, P_{\beta}^{(m)}\right),
$$

where $U \in \mathscr{G}$. Here $U$ has to be embedded in $\mathrm{Sp}(6, R)$ (or $\mathrm{Sp}(4, R)$ if $\mathscr{G} \in \mathrm{SU}(2))$ using the procedure outlined in Appendix D of ref. [5] so that it can act on the phase space functions.

The standard way of constructing an invariant is to use the invariant integral from group theory [9]. For a Lie group $\mathscr{G}$, it is defined as

$$
I=\int_{\mathscr{G}} h(U) \sigma(U) \mathrm{d} U
$$

where $U \in \mathscr{G}, h(U)$ is a function defined on $\mathscr{G}$, and $\sigma(U)$ is the Haar measure for the Lie group $\mathscr{G}$. In our case, the $U$ 's are taken to be the matrices defined in (2.13) or (2.14), depending on whether the group $\mathscr{G}$ is taken to be $\mathrm{SU}(2)$ or $\mathrm{SU}(3)$. Since we are interested in symmetric, positive definite, invariant bilinear forms on the space of homogeneous polynomials of degree $m$, it is natural to take

$$
h(U)=\left[\mathscr{D}^{m}\right]^{T}(U) \mathscr{D}^{m}(U),
$$

where $\mathscr{D}^{m}$ is a $N(2 n, m) \times N(2 n, m)$ matrix defined by

$$
U P_{\alpha}^{m}(z)=P_{\alpha}^{m}(U z)=\sum_{\gamma=1}^{N(2 n, m)} \mathscr{D}^{m}(U)_{\alpha}^{\gamma} P_{\gamma}^{m}(z),
$$

and $\left[\mathscr{D}^{m}\right]^{T}$ is the transpose of $\mathscr{D}^{m}$. From standard matrix theory, we know that $\left[\mathscr{D}^{m}\right]^{T}(U) \mathscr{D}^{m}(U)$ is both symmetric and positive definite. Thus we have an invariant metric on the space of homogeneous polynomial of degree $m$ given as follows:

Pramana - J. Phys., Vol. 58, No. 3, March 2002 


$$
g_{i j}^{(m)}=\left(P_{i}^{(m)}, P_{j}^{(m)}\right)=\int_{\mathscr{G}}\left(\left[\mathscr{D}^{m}\right]^{T}(U) \mathscr{D}^{m}(U)\right)_{i j} \sigma(U) \mathrm{d} U
$$

We now explicitly evaluate the invariant metric $g_{i j}^{(m)}$ given above for various values of $n$ (the number of degrees of freedom) and $m$ (the degree of the homogeneous polynomials). For this, we have to carry out the invariant integration over SU(n) using the appropriate parameterization of the group. The calculations are very laborious and so we do not reproduce them here. We will only summarize the results. Further, we will also list the invariant metric for $m=2$ for the sake of completeness. Since we are interested only in monomials of degree greater than or equal to 2 we take the index $\alpha$ appearing in (2.9) to be

$$
\alpha\left(r_{1}, r_{2}, \ldots, r_{2 n}\right)=i\left(r_{1}, r_{2}, \ldots, r_{2 n}\right)-i(0,0, \ldots, 0,1) .
$$

Since the metric is symmetric, i.e. $g_{i j}^{(m)}=g_{j i}^{(m)} \forall i, j$, it is enough to list down the elements $g_{i j}$ for $i<j$.

\subsection{Two degrees of freedom}

The non-zero elements of the metric $g_{i j}^{(2)}$ for the homogeneous polynomials of degree 2 in two degrees of freedom are as follows:

$$
\begin{aligned}
g_{i i}^{(2)} & =\frac{3}{4}, \quad i \in\{1,5,8,10\}, \\
& =\frac{4}{3}, \quad i \in\{2,3,4,6,7,9\}, \\
g_{i j}^{(2)} & =\frac{1}{12}, \quad \forall i, j \in\{1,5,8,10\} \text { and } i<j .
\end{aligned}
$$

The non-zero elements of the metric $g_{i j}^{(3)}$ for the homogeneous polynomials of degree 3 in two degrees of freedom are

$$
\begin{aligned}
g_{i i}^{(3)} & =\frac{25}{48}, \quad i \in\{11,21,27,30\}, \\
& =\frac{9}{4}, \quad i \in\{16,17,19,25\}, \\
& =\frac{61}{48}, \quad i \in\{12 \ldots 15,18,20,22 \ldots 24,26,28,29\}, \\
g_{i j}^{(3)} & =\frac{7}{48}, \quad \forall i, j \in\{11,15,18,20\} \text { and } i<j, \\
& =\frac{7}{48}, \quad \forall i, j \in\{12,21,24,26\} \text { and } i<j, \\
& =\frac{7}{48}, \quad \forall i, j \in\{13,22,27,29\} \text { and } i<j, \\
& =\frac{7}{48}, \quad \forall i, j \in\{14,23,28,30\} \text { and } i<j .
\end{aligned}
$$


The non-zero elements of the metric $g_{i j}^{(4)}$ for the homogeneous polynomials of degree 4 in two degrees of freedom are

$$
\begin{aligned}
& g_{i i}^{(4)}=\frac{691}{1920}, \quad i \in\{31,51,61,65\}, \\
& =\frac{87}{80}, \quad i \in\{32, \ldots, 34,41,47,50,52,53,57,60,62,64\}, \\
& =\frac{727}{480}, \quad i \in\{35,38,40,54,56,63\} \text {, } \\
& =\frac{631}{240}, \quad i \in\{36,37,39,42, \ldots, 44,46,48,49,55,58,59\}, \\
& =\frac{47}{10}, \quad i \in\{45\} \text {, } \\
& g_{i j}^{(4)}=\frac{169}{960}, \quad i \in\{31\}, j \in\{35,38,40\} ; \quad i \in\{51\}, j \in\{35,54,56\} \text {, } \\
& i \in\{61\}, j \in\{38,54,63\} ; \quad i \in\{65\}, j \in\{40,56,63\}, \\
& g_{i j}^{(4)}=\frac{17}{960}, \quad i \in\{31\}, j \in\{54,56,63\} ; \quad i \in\{51\}, j \in\{38,40,63\} \text {, } \\
& i \in\{61\}, j \in\{35,40,56\} ; \quad i \in\{65\}, j \in\{35,38,54\}, \\
& g_{i j}^{(4)}=\frac{5}{16}, \quad i \in\{32\}, j \in\{41\} ; \quad i \in\{33\}, j \in\{47\} ; i \in\{34\}, j \in\{50\}, \\
& i \in\{52\}, j \in\{57\} ; i \in\{53\}, j \in\{60\} ; i \in\{62\}, j \in\{64\}, \\
& g_{i j}^{(4)}=\frac{19}{60}, \quad i \in\{32,41\}, j \in\{44,46\} ; \quad i \in\{33,47\}, j \in\{42,49\}, \\
& i \in\{34,50\}, j \in\{43,48\}, \quad i \in\{36,59\}, j \in\{52,57\}, \\
& i \in\{37,58\}, j \in\{53,60\} ; \quad i \in\{39,55\}, j \in\{62,64\} \text {, } \\
& g_{i j}^{(4)}=\frac{1}{240}, \quad i \in\{32\}, j \in\{64\} ; \quad i \in\{33\}, j \in\{53\} ; i \in\{34\}, j \in\{57\}, \\
& i \in\{41\}, j \in\{62\} ; i \in\{47\}, j \in\{60\} ; \quad i \in\{50\}, j \in\{52\}, \\
& g_{i j}^{(4)}=-\frac{1}{240}, \quad i \in\{32\}, j \in\{62\} ; \quad i \in\{33\}, j \in\{60\} ; i \in\{34\}, j \in\{52\}, \\
& i \in\{41\}, j \in\{64\} ; \quad i \in\{47\}, j \in\{53\} ; \quad i \in\{50\}, j \in\{57\}, \\
& g_{i j}^{(4)}=\frac{1}{80}, \quad i \in\{36\}, j \in\{48\} ; \quad i \in\{37\}, j \in\{42\} ; i \in\{39\}, j \in\{46\}, \\
& i \in\{55\}, j \in\{44\} ; \quad i \in\{58\}, j \in\{49\} ; \quad i \in\{59\}, j \in\{43\}, \\
& g_{i j}^{(4)}=-\frac{1}{80}, \quad i \in\{36\}, j \in\{43\} ; \quad i \in\{37\}, j \in\{49\} ; i \in\{39\}, j \in\{44\} \text {, } \\
& i \in\{55\}, j \in\{46\} ; i \in\{58\}, j \in\{42\} ; i \in\{59\}, j \in\{48\} \text {, } \\
& g_{i j}^{(4)}=\frac{73}{240}, \quad i \in\{36\}, j \in\{59\} ; \quad i \in\{37\}, j \in\{58\} ; i \in\{39\}, j \in\{55\}, \\
& i \in\{42\}, j \in\{49\} ; \quad i \in\{43\}, j \in\{48\} ; i \in\{44\}, j \in\{46\},
\end{aligned}
$$




$$
\begin{array}{ll}
g_{i j}^{(4)}=\frac{31}{160}, \quad & i \in\{35\}, j \in\{38,40,54,56\} ; \quad i \in\{38\}, j \in\{54,63\}, \\
& i \in\{40\}, j \in\{38,56,63\} ; \quad i \in\{54\}, j \in\{56,63\} ; \\
& i \in\{56\}, j \in\{63\}, \\
g_{i j}^{(4)}=\frac{23}{480}, \quad & i \in\{35\}, j \in\{63\} ; \quad i \in\{38\}, j \in\{56\} ; \quad i \in\{40\}, j \in\{54\}, \\
g_{i j}^{(4)}=\frac{19}{1920}, \quad & \forall i, j \in\{31,51,61,65\} \text { and } i<j .
\end{array}
$$

\subsection{Three degrees of freedom}

The non-zero elements of the metric $g_{i j}^{(2)}$ for the homogeneous polynomials of degree 2 in three degrees of freedom are

$$
\begin{aligned}
g_{i i}^{(2)} & =\frac{11}{16}, \quad i \in\{1,7,12,16,19,21\} \\
& =\frac{5}{4}, \quad i \in\{2, \ldots, 6,8, \ldots, 11,13, \ldots, 15,17,18,20\}, \\
g_{i j}^{(2)} & =\frac{1}{16}, \quad \forall i, j \in\{1,7,12,16,19,21\} \text { and } i<j .
\end{aligned}
$$

The non-zero elements of the metric $g_{i j}^{(3)}$ for the homogeneous polynomials of degree 3 in three degrees of freedom are

$$
\begin{aligned}
& g_{i i}^{(3)}= \frac{5}{12}, \quad i \in\{22,43,58,68,74,77\}, \\
&= \frac{21}{20}, \quad i \in\{23, \ldots, 28,33,37,40,42,44,45, \ldots, 48,52,55,57, \\
&59, \ldots, 62,65,67,69,70,71,73,75,76\}, \\
&= \frac{19}{10}, \quad i \in\{29, \ldots, 32,34,35,36,38,39,41,49,50,51,53,54, \\
&\quad 56,63,64,66,72\}, \\
& g_{i j}^{(3)}=\frac{1}{10}, \quad \forall i, j \in\{22,28,33,37,40,42\} \text { and } i<j, \\
&=\frac{1}{10}, \quad \forall i, j \in\{23,43,48,52,55,57\} \text { and } i<j, \\
&=\frac{1}{10}, \quad \forall i, j \in\{24,44,58,62,65,67\} \text { and } i<j, \\
&=\frac{1}{10}, \quad \forall i, j \in\{25,45,59,68,71,73\} \text { and } i<j, \\
&=\frac{1}{10}, \quad \forall i, j \in\{26,46,60,69,74,76\} \text { and } i<j, \\
&=\frac{1}{10}, \quad \forall i, j \in\{27,47,61,70,75,77\} \text { and } i<j .
\end{aligned}
$$




\section{Invariant norm}

We are now in a position to obtain the invariant norm. Consider the symplectic map $\mathscr{M}$ describing the Hamiltonian system. As discussed in the introduction, we retain only the leading nonlinear term and get

$$
\mathscr{M}=\hat{M} \mathrm{e}^{: f_{3}} \text {. }
$$

Next we transform this map into its normal form and then write out the invariant norm for $f_{3}$ in the transformed phase space coordinates.

We now give details of the calculations outlined above. Let $A$ be the symplectic transformation that takes $M$ into its normal form $N$. Then the normal form $\mathscr{N}$ of the symplectic map $\mathscr{M}$ is given by

$$
\mathscr{N}=A^{-1} \mathscr{M} A=A^{-1} M A A^{-1} \mathrm{e}^{:} f_{3}: A=N \mathrm{e}^{:} f_{3}^{\mathrm{rr}},
$$

where

$$
f_{3}^{\mathrm{tr}}=A^{-1} f_{3}(z)=f_{3}\left(A^{-1} z\right) \text {. }
$$

Since $A$ depends on $M, f_{3}$ also now depends on $M$. The homogeneous polynomial $f_{3}^{\operatorname{tr}}$ can be expressed as a linear combination of the basis monomials as follows.

$$
f_{3}^{\mathrm{tr}}(z)=b_{\alpha} P_{\alpha}^{(3)}(z)
$$

The quantities $b_{\alpha}$ are real constants.

We are now in a position to define the invariant norm which is given by

$$
I(z)=\left(f_{3}^{\mathrm{tr}}(z), f_{3}^{\mathrm{tr}}(z)\right)^{1 / 2}
$$

where the symmetric, positive definite, invariant, bilinear form $(\cdot, \cdot)$ is defined in (3.20) through the basis monomials with $m=3$. This is invariant under the action of $\mathscr{N}$. Using the expansion of $f_{3}^{\text {tr }}(z)$ in terms of the basis monomials we obtain

$$
\begin{aligned}
\left(f_{3}^{\mathrm{tr}}(z), f_{3}^{\mathrm{tr}}(z)\right) & =\left(\sum_{\alpha=1}^{N(2 n, 3)} b_{\alpha} P_{\alpha}^{(3)}(z), \sum_{\beta=1}^{N(2 n, 3)} b_{\beta} P_{\beta}^{(3)}(z)\right), \\
& =\sum_{\alpha=1}^{N(2 n, 3)} \sum_{\beta=1}^{N(2 n, 3)} b_{\alpha} b_{\beta}\left(P_{\alpha}^{(3)}(z), P_{\beta}^{(3)}(z)\right), \\
& =\sum_{\alpha=1}^{N(2 n, 3)} \sum_{\beta=1}^{N(2 n, 3)} b_{\alpha} b_{\beta} g_{\alpha \beta}^{(3)} .
\end{aligned}
$$

Here the elements of $g_{\alpha \beta}^{(3)}$ are given in (3.23) and (3.25) for $n=2$ and $n=3$ respectively. Since $g_{\alpha \beta}^{(3)}$ is an invariant metric, $I(z)$ is a well-defined invariant norm.

Notice that $I(z)$ is a function of the coefficients $b_{\alpha}$ which in turn are related to the coefficients $a_{\alpha}$ which parameterize the symplectic map $\mathscr{M}$ (and hence the original Hamiltonian system). Moreover, $I(z)$ is a polynomial of degree 2 which quantifies the leading nonlinearity of the system. Therefore, one can attempt to vary one of the parameters describing the original system and minimize the norm $I(z)$. The system performance with this optimal parameter value would be enhanced since the nonlinearity is reduced. We demonstrate this in the final section using an example. 


\section{Application}

In the previous section, we have defined a norm $I(z)$ using $f_{3}^{\text {tr }}$ of the symplectic map in its normal form. Further, we argued that minimizing this by varying one of the system parameters should enhance the system performance. We now demonstrate this through an example. In this case, the region of stability of the system is shown to increase significantly.

For example, consider a particle being transported through the following 'FODO Cell' (a common component of a particle accelerator) which consists of a thin lens corrector, a drift, a focusing quadrupole, a drift, a thin lens corrector, a drift, a defocusing quadrupole, a drift and another thin lens corrector in the given order. We will show that the stability of the system can be enhanced by minimizing the norm as a function of the length-strength product $s$ of the thin lens correctors.

We will consider the case where the first and the last have the same strength $s_{1}$ and the middle corrector has a different strength $s_{2}$. The quadrupole strength is set at 0.01 and the length of the drift is taken to be $0.25 \mathrm{~m}$. Since we restrict ourselves to $f_{3}$, the drift has only a linear part. The individual map elements for the $\operatorname{drift}\left(M_{\mathrm{d}}\right)$, the focusing quadrupole $\left(M_{\mathrm{f}}\right.$, $\left.f_{3}^{(\mathrm{f})}\right)$ and the defocusing quadrupole $\left(M_{\mathrm{df}}, f_{3}^{(\mathrm{df})}\right)$ are as follows:

$$
\begin{aligned}
& M_{\mathrm{d}}=\left(\begin{array}{cccc}
1 & 0.25 & 0 & 0 \\
0 & 1 & 0 & 0 \\
0 & 0 & 1 & 0.25 \\
0 & 0 & 0 & 1
\end{array}\right) \\
& M_{\mathrm{f}}=\left(\begin{array}{cccc}
0.379702 & 0.783113 & 0 & 0 \\
-1.09285 & 0.379702 & 0 & 0 \\
0 & 0 & 1.78278 & 1.24937 \\
0 & 0 & 1.74352 & 1.78278
\end{array}\right) \\
& f_{3}^{(\mathrm{f})}=0.000729027786268741 p_{1}{ }^{3}-0.00401370331668622 p_{1} p_{2}{ }^{2} \\
& -0.00223402941215277 p_{1}^{2} q_{1}+0.00366214590673008 p_{2}^{2} q_{1} \\
& +0.00315085652798429 p_{1} q_{1}^{2}-0.00260362598064677 q_{1}^{3} \\
& +0.013396336186578 p_{1} p_{2} q_{2}-0.013872226822993 p_{2} q_{1} q_{2} \\
& -0.011804190022307 p_{1} q_{2}^{2}+0.016039119622458 q_{1} q_{2}^{2} \text {, } \\
& M_{\mathrm{df}}=\left(\begin{array}{cccc}
1.78278 & 1.24937 & 0 & 0 \\
1.74352 & 1.78278 & 0 & 0 \\
0 & 0 & 0.379702 & 0.783113 \\
0 & 0 & -1.09285 & 0.379702
\end{array}\right) \\
& f_{3}^{(\mathrm{df})}=0.00184546878109027 p_{1}{ }^{3}-0.00294431866040622 p_{1} p_{2}{ }^{2} \\
& -0.0090716074824943 p_{1}^{2} q_{1}+0.00501551187521087 p_{2}^{2} q_{1} \\
& +0.015553938120045 p_{1} q_{1}{ }^{2}-0.010031600023799 q_{1}{ }^{3} \\
& +0.00567688973384745 p_{1} p_{2} q_{2}-0.011148606961365 p_{2} q_{1} q_{2} \\
& -0.00371890079800577 p_{1} q_{2}{ }^{2}+0.010435916746424 q_{1} q_{2}^{2} \text {. }
\end{aligned}
$$


The map $f_{3}^{(\mathrm{c})}$ for a corrector is

$$
f_{3}^{(\mathrm{c})}=-0.465174\left(q_{1}^{3}-3 q_{1} q_{2}^{2}\right) s,
$$

where $s$ is the length-strength product that has to be minimized. In our case, we will have two different maps - one for the end correctors (depending on $s_{1}$ ) and another for the middle corrector (depending on $s_{2}$ ).

Using CBH theorem from Lie group theory [9] we combine the maps of all individual elements to get one single map describing the entire FODO cell. The final combined matrix $M$ is given by

$$
M=\left(\begin{array}{cccc}
-2.22235 & 2.24671 & 0.0 & 0.0 \\
-2.239 & 1.81356 & 0.0 & 0.0 \\
0.0 & 0.0 & 1.81356 & 2.24671 \\
0.0 & 0.0 & -2.239 & -2.22235
\end{array}\right)
$$

The expression for the combined third degree polynomial $f_{3}$ is very long and is hence not given here.

We now put the map in normal form. The matrix $A$ which puts the map $\mathscr{M}$ in the normal form and the normal form $N$ are given below

$$
\begin{aligned}
A & =\left(\begin{array}{cccc}
1.51498 & 0 & 0.0 & 0.0 \\
1.36073 & 0.660076 & 0.0 & 0.0 \\
0.0 & 0.0 & 1.51498 & 0 \\
0.0 & 0.0 & -1.36073 & 0.660076
\end{array}\right), \\
N & =\left(\begin{array}{cccc}
-0.204398 & 0.97889 & 0.0 & 0.0 \\
-0.978887 & -0.204397 & 0.0 & 0.0 \\
0.0 & 0.0 & -0.204397 & 0.97889 \\
0.0 & 0.0 & -0.978887 & -0.204398
\end{array}\right) .
\end{aligned}
$$

The polynomial $f_{3}$ also gets transformed to $f_{3}^{\text {tr }}$ by this process. We now calculate the norm $I(z)$ using this transformed $f_{3}^{\text {tr }}$ and minimize it as a function of $s_{1}$ and $s_{2}$.

The minimum value for $I(z)$ is obtained when the corrector strengths are given as follows:

$$
s_{1}=-0.00134675 ; \quad s_{2}=-0.0269976 .
$$

The boundary of the region of stability (dynamic aperture) of the system is found using the solvable map method [11]. Along the $q_{1}$ direction, the dynamic aperture is found to be

1. $\left(q_{1}, p_{1}, q_{2}, p_{2}\right)=(0.18,0,0,0)$ when all correctors are turned off.

2. $\left(q_{1}, p_{1}, q_{2}, p_{2}\right)=(2.2,0,0,0)$ when the correctors are turned on with the above strengths.

Similar results are observed in the other directions. We notice that the dynamic aperture has increased by an order of magnitude.

\section{Conclusions}

We have defined an invariant metric on the space of homogeneous polynomials characterizing a symplectic map. We have demonstrated that the metric thus defined helps in 
improving the stability and hence the performance of the system. This then promises to be a handy tool that can be employed to enhance the performance of nonlinear Hamiltonian systems.

\section{Acknowledgement}

This work was supported by research grants from ISRO and DRDO through the Nonlinear Studies Group of IISc. This work was also supported by Homi Bhabha Fellowship.

\section{References}

[1] A J Dragt, Physics of High Energy Particle Accelerators in AIP Conference Proceedings No. 87, edited by R A Carrigan, F Huson and M Month (American Institute of Physics, New York, 1982) 147

A J Dragt, F Neri, G Rangarajan, D R Douglas, L M Healy and R D Ryne, Ann. Rev. Nucl. Part. Sci. 38, 455 (1988) and references therein

[2] A J Dragt, E Forest and K B Wolf, in Lie methods in optics edited by J S Mondragon and K B Wolf (Springer Verlag, Berlin, 1986)

[3] V Lakshminarayanan, R Sridhar and R Jagannathan, J. Opt. Soc. Am. A15, 2497 (1998)

[4] G Rangarajan, Invariants for symplectic maps and symplectic completion of symplectic jets Ph.D. thesis (University of Maryland, 1990)

[5] G Rangarajan, J. Math. Phys. 37, 4514 (1996)

[6] A J Dragt and J M Finn, J. Math. Phys. 17, 2215 (1976)

[7] A Nijenhuis and H S Wilf, Computational algorithms for computers and calculators (Academic Press, New York, 1978)

[8] A Giorgilli, Comp. Phys. Comm. 16, 331 (1979)

[9] J F Cornwell, Group theory in physics (Academic Press, New York, 1984) vols. I and II

[10] G Guralnik, T Warnock and C Zemach, J. Comp. Phys. 61, 89 (1985)

[11] G Rangarajan and M Sachidanand, J. Phys. A33, 131 (2000) 